times at 164, oco feet (NATURE, vol. li. p. 534). If these layers tapered off uniformly in either direction from the region of maximum deposit, the total mean thickness would be half this, or $\$ 2,000$ feet; and if the mean rate of subsidence were never greater than 2.18 feet per century, the total time required for the accumulation of Cambrian and post-Cambrian rocks would be not less than $3 \frac{3}{4}$ millions of years. But there may have been long unknown gaps in the process of their accumulation; the outer margin of the deposits may have extended far beyond the area of subsidence, and the mean rate of subsidence may have been at all times considerably less than the upper limit given above. On these accounts, as well as on others that might be mentioned, it seems possible that much more than $3 \frac{3}{4}$ million years has elapsed since the beginning of the Cambrian period.

Birmingham, April 8.

C. Davison.

\section{The Burmese Chipped Flints Pliocene not Miocene.}

IN the Geological Magazine for November of last year, p. 525 , is a review by Prof. T. Rupert Jones, of the imporiant paper, published in the Records of the Geological Survey of India, by Dr. Fritz Noetling, the Palæontologist of the Survey, "On the occurrence of Chipped (?) Flints in the Upper Miocene of Burma." Another paper, by Prof. T. R. Jones, on "Miocene Man in India," appeared in Natural Science for the same month.

From the fact that the mammals Rhinoceros perimensis and Hipparion antelopinum, of which bones were found associated with the flint chips, have only been found in India in Pliocene beds, and from a slight acquaintance, gained, it is true, more than thirty years ago, with the Burmese strata in which Dr. Noetling's most interesting discovery was made, I felt assured that there must be some error in believing that the flint chips occurred in Miocene deposits, and I wrote to Dr. Noetling on the subject. I have just heard from him in reply. In a lette from Upper Burma of March XI, he tells me he has now de finitely ascertained that the bed containing the chipped ffints is Pliocene.

Further particulars will, I hope, be published before long by Dr. Noetling; and I should not have written on the subject but that a serious error is caused by its being supposed that "Miocene Man" has been shown to have existed in India, and it is desirable that this error should be corrected without delay. The importance of the discovery is in no way diminished by the correction of the geological date to which the flint-bearin stratum is referred.

W. T. BLANFORD.

April 17.

\section{The Mandrake.}

WITH regard to Prof. Veth's exhaustive account of the mandrake (referred to in NATURE of April 11, p. 573), it may be useful to students of folklore to call their attention to the occurrence in the Chinese literature of a similar superstition, wherein Phytolacca acinosa (Shangluh) takes the place of Mandragora officinurum. Sie Tsai-Kang's "Wu-tsah-tsu," written about 1610 (Japanese edition, I66I, tome x. p. 4I), contains the following passage :"The Shang-luh grows on the ground beneath which dead man lies; hence its root is mostly shaped like a man. ${ }^{3}$. . . In a calm night when nobody is about, the collector, offering the owl's flesh roasted with oil, propitiates the spirit of the plant until ignes fatui crowd about the latter; then the root is dug out, brought home and prepared with magic paper for a week; thus it is made capable of speech. This plant is surnamed 'Ye-hu' (i.e. Night Cry) on account of its demoniacal nature. ${ }^{2}$ There are two varieties of it: the white one is used for medicine ; the red one commands evil spirits, and kills men when it is internally taken by error." KUMAGUSU MINAKATA. April 16.

1 Here the author says: "It is popularly called "Chang-liu-Kan' (=Witch-tree-root)." The name shows that the root was used in witchcraft, similarly with that of the Mandragora ( $c f$. Hone, "The Year-Book," sub. "December 28 ")

2 Another explanation suggested for this name is that, as long as the fruit of the Phytolacca remains unripe, the cuckoo continues to cry every night

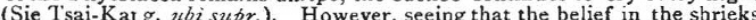
of the Mandragora was once current among the Europeans ("Encyclo. of the Mandragora was once current among the Europeans ("Encyclothe Chinese name "Night Cry" from an analogous origin.

No. [33O, vol. $5 \mathrm{I}]$

\section{A Claim for Priority.}

I SEND you, under separate cover, a copy of an address, "Radiant Matter," \&c., delivered at the International Electrical Exhibition, held in Philadelphia in 1884 , reprinted from the Journal of the Franklin Institute, September 1885 , and would call your attention to the description of the method of preparing films of gold and other metals of extreme thinness, far exceeding in tenuity those described in NATURE as novelties in metallurgical methods (prepared in identically the same manner), and exhibited at a conversazione of the Royal Society, June I 3 , I894. The first published note regarding this subject may be found in the Proceedings of the American Phil. Soc., vol. xcix. February 16, 1877. Later and fuller notices will be found in fourn. Franklin Institute, April 1877, June 1877, September 1885, and September 1894. In addition to the above, the process was fully described in U.S. Patent, 198, 209, December 18,1877 .

Philadelphia, April 5.

Alex. E. OUterbridge.

\section{AN IMPROVED METHOD FOR THE MICRO} SCOPIC INVESTIGATION OF CRYSTALS.

A MEMOIR of considerable importance to all who are interested in the microscopic determination of the characters of crystals, is contributed by Prof. Klein to the Sitzungsberichte of the Berlin Akademie der Wissenschaften for January 3I, I 895. The two essential points of the communication are that a form of stage goniometer is described, which permits of the most complete examination of many of the principal zones of the crystal with one and the same setting of the crystal upon its holder, and that the crystal is immersed during the observations in a liquid whose refractive index is about the mean of the refractive indices of the crystal. The idea of the "Universaldrehapparat," as the new stage goniometer is termed, appears to have suggested itself almost simultaneously to Prof. Klein and to Herr von Federow, for the former described an earlier form of it in the Sitzungsberichte of April I89I, while the latter published a description of an "Universaltischen" for the microscope in the Zeitschrift für Krystallographie of May in the same year. Herr von Federow had previously contributed to the Zeitschrift a remarkable memoir concerning a theodolitic universal goniometer, and the application of the principle of that instrument to the microscope goniometer followed naturally therefrom. The present memoir of Prof. Klein affords so admirable a description of the improved instrument, which has been constructed for him by the well-known Berlin crystallographical optician, Herr Fuess, and likewise of the mode of employing it in connection with the immersion method, that readers of NATURE may find a brief account of it not uninteresting. Unfortunately this description cannot well be illustrated, as Prof. Klein's illustrations are photographic reproductions which are unsuitable for further reproduction.

The microscope should of course be one of the petrological type, fitted with the usual accessories for the examination of crystals in parallel and convergent polarised light. The particular instrument constructed for Prof. Klein is somewhat similar to the largest Fuess model. It is so arranged with respect to the centre of gravity that it can be rotated into the horizontal position whenever desired, a point of some importance with regard to the use of an immersion liquid. The stage is of course circular, and is divided so as to read with the aid of a pair of verniers to single minutes; it is further provided above with two graduated rectangular traversing movements, one of which is supplied with a micrometer registering $0.01 \mathrm{~m} . \mathrm{m}$., while the other is capable of much more rapid motion. The advantages of the simultaneous rotation of the polarising and analysing nicols, as adopted in the microscopes made by Mr. Swift under the direction of Mr. Allan Dick, have been so well appreciated by 
Prof. Klein, that this has been arranged for in the new Fuess instrument. The carriers of the nicols are each furnished with a toothed flange capable of gearing with a smaller pinion, and the two pinions are arranged at the ends of a connecting rod furnished at a convenient height near the upper pinion with a milled flange by means of which rotation can be effected. Provision is made for the lengthening of the connecting rod when the focussing of the microscope by the rack and pinion or by the fine adjustment is effected, and care is also taken that the rotation by means of the connecting rod shall occur without dead-space or backlash. Prof. Klein sta tes that some important details in connection with improvements in the mode of carrying out this simultaneous movement of polariser and analyser will shortly be published by Herr Fuess. Provision has likewise been made for correcting at any time the setting of the nicols in their carriers, experience having shown that the setting invariably alters slightly in course of time. In addition to the eyepiece nicol capable of being connected with the polariser in the manner just described, there is likewise provided the usual nicol capable of sliding in or out of the microscope tube just over the objective. Above this, and just below the eyepiece, a Bertrand lens for observing interference figures in convergent light is capable of sliding in and out of the tube, and is intended to be employed in conjunction with a converging system of lenses capable of being carried in a tube attachment beneath the level of the stage. The remaining details of the microscope are the same as are usually supplied with the No. I Fuess instrument.

The stage goniometer is intended to be employed with the microscope arranged horizontally, as it is found inconvenient to employ an immersion liquid with a vertical arrangement. The base-plate of the goniometer, consisting of a stout metal plate with fairly large central aperture, is fixed by a suitable clamping arrangement upon the now vertical stage of the microscope. The plate is continued into a short arm on that side which is uppermost when fixed in position, and this arm carries near its end, and at right angles to it (horizontal when in position), a projecting piece terminating in the supporting cone for the goniometer circle, and which also carries the vernier reading to five minutes and the fine adjustment. The circle is hollowed in its upper central part, and perforated with a central aperture; this permits of the sliding movement within the hollow, for centering purposes, of a disc which carries the axis of the instrument. To the lower end of this short axis are attached the movements for adjusting the crystal, and the lower of which carries the crystal. The adjusting movements are a pair of circular quadrants arranged at right angles to each other and graduated. They are simpler in construction, and lie much closer together than those of the best forms of goniometer now in use for ordinary goniometric and spectrometric work, and are therefore particularly suitable for use in connection with the microscope. The upper quadrant is fixed to the axis ; over it a slider is capable of moving, which carries a vernier, and below it the lower quadrant, which in turn is fitted with a slider terminating in the holder which carries the crystal cemented by wax. The verniers enable readings of five minutes to be obtained, the same degree of accuracy as in the case of the circle.

The glass cell containing the immersion liquid is supported in position normal to the axis of the microscope by means of a stand with an adjustable arm placed to the left of the microscope. It is recommended to have a series of cells, ready for filling with various media of the most frequently required refractive power. The advantages of Adams' method of determining optic axial angles may also be combined with those of the method now described, by use of a cell consisting of an upper cylindrical portion terminating below in a sphere filled with the liquid. As regards suitable liquids, an admirable list is given by Herr Pulfrich in his book descriptive of the construction and use of the total-reflectometer recently devised by him (p. 64). Two errors in that list, however, are corrected by Prof. Klein; he has been unable to prepare the solution of mercuric iodide in aniline and quinoline of refractive index $2 \cdot 2$, and the refractive index of the phenyl sulphide kindly supplied by Prof. Klein's colleague, Prof. Emil Fischer, is only $1{ }^{\circ} 6$ instead of $\mathrm{I} \cdot 95$. If the dangerously poisonous and inflammable liquids are excluded, the list consists chiefly of oils, the well-known 'Thoulet solution, monobromnaphthalene, and methylene iodide. The solution of iodine in the latter frequently renders it insufficiently transparent for the purpose.

The determination of the true angle, $2 \mathrm{~V}$, between the optic axes within the crystal, supposing it to be biaxial, can at once be determined with the aid of the new apparatus, by immersing the crystal in a liquid whose refractive index is equal to the $\beta$ (the intermediate) refractive index of the crystal. The condensing system of lenses is first inserted between the polarising nicol and the stage, and the Bertrand lens above the analyser; as objective, either the ordinary wide angle combination usually employed for convergent light work, or a specially constructed one supplied for the particular purpose of convergent light observations through an immersion liquid, is employed. This objective is so constituted that as large a field of vision as possible is afforded, while the distance between objective and crystal is considerably greater than with the ordinary systems in use. The apparent angle of the optic axes in air, $2 \mathrm{E}$, may first be measured, if desired, after adjustment of the crystal by means of the adjusting movements, by bringing the hyperbolic brushes to the cross wire of the microscope eyepiece in the usual manner. The immersion cell not being in position while this is being achieved, the objective can be approached nearer to the crystal and one of the ordinary forms of convergent light objective employed, which affords a larger angle of vision, reserving the special objective for the determination of the true angle of the optic axes. If, however, the Adams spherical cell is employed, there is no necessity even here to use the special objective, as the older wide angle form serves admirably. With the parallel sided cells it is preferable to use the special objective. The Adams sphere is not supported similarly to the rectangtilar cells, but is conveniently held by its cylindrical neck in a small support directly attached to the lower quadrant of the adjusting apparatus. The measurement of the true angle of the optic axes is then carried out in the usual manner, similarly to the determination of the apparent angle in air, while the crystal is immersed in the liquid contained in one or other of the two forms of cell. Monochromatic light should of course be used in making the observations, a sodium flame some little distance in front of the polariser being employed by Prof. Klein.

The great advantage of this method of determining the true inner angle between the optic axes lies in the fact that it is totally unnecessary to prepare sectionplates of the crystal, the whole crystal itself being employed, and thus material saved. Prof. Klein does not claim for it the highest attainable accuracy, and for the class of work such as that with which the writer of this article has become identified, the determination of the crystallographic characters of series of isomorphous compounds closely resembling each other, where every endeavour must be made to attain the upper limits of experimental accuracy, such a method is of course inadequate. But for the ordinary description of minerals and the crystals of isolated chemical preparations unlikely to be injured by the immersion liquid, and particularly for laboratory teaching, the method is one of 
the simplest and most interesting yet described. The accuracy depends entirely upon the closeness of the approximation of the refractive index of the liquid to the $\beta$ index of the crystal. Of course it will rarely happen that coincidence of these values will occur for all colours of the light employed, the dispersion of the crystal and the liquid in general being different. So that although the values may be coincident for sodium light, they would in all probability be different for other colours. But if the observations are only conducted for sodium light, a process which is frequently sufficient for the purpose in view, then this objection entirely disappears. Moreover, the errors introduced by the discrepancy for different wave-lengths of light would not be sufficiently large in most cases, if observations for other colours were made, to materially reduce the value of the method for the purposes for which it was designed.

A consideration of the simple formulæ connecting the optic axial angle with the $\beta$ refractive index and the refractive index of an immersion liquid will at once render the value of the method, within the above specified limits, clear. Representing as usual the real semiacute angle between the optic axes within the crystal by $\mathrm{Va}$, the semi-obtuse angle by Vo, and the apparent semiacute and obtuse angles in the immersion liquid by $\mathrm{Ha}$ and Ho respectively, the refractive index of the medium for light of the same wave-length being $n$, then :

$$
\mathrm{Sin} \mathrm{Va}=\frac{n}{\beta} \sin \mathrm{Ha} \text { and } \sin \mathrm{Vo}=\frac{n}{\beta} \sin \mathrm{Ho} .
$$

These two equations are of the same kind, for both $\mathrm{Va}$ and Vo are less than $90^{\circ}$; and the only variables are $n$ sin $\mathrm{Ha}$ and $n$ sin $\mathrm{Ho}$, for $\beta$, sin $\mathrm{Va}$, and $\sin \mathrm{Vo}$ are constant quantities for this wave-length of light. If, now, the sum of the angles $2 \mathrm{Ha}$ and $2 \mathrm{Ho}$ is greater than $180^{\circ}$, the common factor $n$ must, in order to bring the sum of these angles down equal to $180^{\circ}$, be increased, that is, a liquid of higher refractive power be employed. Conversely if the sum is less than $180^{\circ}$ the refractive power of the liquid must be diminished in order to bring the sum of the angles up to $180^{\circ}$. For the specially interesting intermediate case where $n=\beta$, the sum of $2 \mathrm{Ha}$ and $2 \mathrm{Ho}$ will be exactly $180^{\circ}$, and $\sin \mathrm{Va}=\sin \mathrm{Ha}$ and $\sin$ $\mathrm{Vo}=$ sin Ho, when also $\mathrm{Va}=\mathrm{Ha}$ and $\mathrm{Vo}=\mathrm{Ho}$.

From the above theoretical considerations one can immediately deduce the course to be taken to render the immersion liquid exactly equal to the $\beta$ index of the crystal; if the measured values of $2 \mathrm{Ha}$ and $2 \mathrm{Ho}$ add up to over $180^{\circ}$ a liquid of higher refraction must be obtained, and vice versa if the sum is less than $180^{\circ}$. There are, however, several ways of determining the closeness of approximation of the indices without going to the trouble of actually making preliminary measurements. In the first place the crystal will disappear in the liquid, that is to say, will be invisible, provided that it is colourless, when its refractive power is equal to that of the surrounding medium, especially when the line of the observer's vision lies in the plane of the optic axes. This is very beautifully observed when calcite is immersed in monobromnaphthaline, and particularly when it is arranged so that the observer looks along the direction of the vertical axis of the crystal; under these conditions the latter is completely invisible. In the second place, instead of hyperbolic curves passing through the positions occupied by the optic axes, the brushes will take the form of almost straight lines when the refraction of crystal and liquid is about the same.

In choosing crystals for observation by the new method, Prof. Klein recommends that individuals or fragments should be selected which are equally thick in two perpendicular directions in the plane of the optic axes, that is, such as are almost cylindrical in appearance, and not too thick to prevent the interference figures being observed. When immersed in the liquid, it is as if at each moment, and for every position during rotation of the crystal, a parallel section-plate were being examined, the natural faces of the crystal-however rich in faces the zone may be-not entering into consideration whatever.

The adiantages of the use of an immersion liquid of equal refractive power in the examination of crystals have been pointed out by several previous observers, as Prof. Klein is careful to state. So long ago as I84I Biot, in his memoir concerning lamellar polarisation, describes the use he made of it. The method has long remained dormant, however, as far as is known from the literature of this branch of study. In the eighth edition, however, of the Lehrbuch der Physik und Meteorologie of Joh. Müller, edited by L. Pfaundler in I879, it is stated that if the refractive index of the liquid in which a plate perpendicular to one of the medium lines is immersed is equal to that of the crystal, the true angle between the optic axes is at once afforded. Latterly, however, the evident advantages of the method have suggested themselves to several crystallographers. M. Fouqué mentions it in his memoir in the Bulletin of the French Mineralogical Society of 1894 on the felspars.

The writer of this article has frequently made use of the method for certain specific purposes, and it may be of use to other workers to give a brief indication of one or two modes of extending its sphere of usefulness not touched upon by Prof. Klein. In the course of the in' estigation of the norral sulphates of potassium, rubidium, and cæsium, the results of which were laid before the Chemical Society last year (Journ. Chem. Soc. 1894 628 , and Zeitschrift für Krystallographie, I 894, xxiv. I), a difficulty was found in determining the true optic axial angle of rubidium sulphate by means of the very accurately orientated section-plates prepared by use of the new grinding goniometer described to the Royal Society (Phil. Trans. 1894, Series A, 887) earlier in the same year. The difficulty, which is one not uncommonly met with, was owing to the fact that the extremely low double refractirn, necessitating the use of very thick sectionplates, combined with the slight separation of the optic axes, rendered it impossible to measure the obtuse angle in monobromnaphthaline, and so to calculate the true angle by means of the formula $\tan \mathrm{Va}=\frac{\mathrm{Sin} \mathrm{Ha}}{\operatorname{Sin} \mathrm{Ho}}$

The

difficulty was surmounted, as fully described in the memoir referred to, by measuring the acute angle by means of section-plates perpendicular to the first median line immersed successively in two liquids, benzene and cedar oil, whose refractive indices were nearly, and the mean of them exactly, equal to the mean refractive index of rubidium sulphate. The two series of values obtained for six wave-lengths of light (the monochromatic light producer recently described by the writer, Phil. Trans. 1894, Series A, 913, being employed) were almost identical, differing only by a very few minutes, and the mean for each wave-length was taken as representing the true angle of separation of the optic axes for that particular wave-length. The method is applicable to all cases where it is found impossible to see the hyperbolic brushes through a section perpendicular to the second median line on account of the slight separation of the optic axes. The suggestion to employ it was made to the writer by Mr. Miers, of the British Museum, who has had a goniometer constructed for the express purpose of studying the use of an immersion liquid.

Another case in which observations in such a liquid are of great value is when it is found desirable to confirm, in some independent manner, the mode of dispersion of the optic axes for different colours indicated by the calculated values of $2 \mathrm{Va}$ obtained from the formula last quoted. Several of the compounds which the writer has lately been engaged in studying exhibit very low dispersion of the optic axes, and the calculated values of NO. 1330, VOL. 51$]$ 
2 Va for five wave-lengths, obtained from the measurements of the apparent acute and obtuse angles in monobromnaphthalene by the use of accurately orientated section-plates, are so close together that it was considered advisable to ascertain in some other manner whether the order of dispersion was truly represented; that is, whether the angle for one end of the spectrum was really very slightly greater than that for the other end, or whether the amount of dispersion thus indicated did not really fall within the limits of experimental error, thus leaving it possible that the dispersion might even be of the contrary order. By immersing a plate perpendicular to the first median line in a liquid of refractive power equal to the medium refractive index of the crystal, the interference figure in white light usually at once indicates, by the colours exhibited on the margins of the axial brushes, the order of dispersion, and measurements of the axial angle for the two extreme wavelengths afford an immediate check upon the accuracy of the calculated angles. It is a considerable source of satisfaction to be able to confirm such calculated optic axial angles in so simple a manner.

Prof. Klein further describes how admirably the new apparatus is adapted for the determination of the extinction angles upon the various faces of a zone, in parallel polarised light. For this purpose the converging ienses are removed, and the eyepiece analysing nicol is employed, so that the polarising and analysing nicols may be arranged for simultaneous rotation. The measurements are carried out while the crystal is immersed in the liquid as in case of the determinations of optic axial angle. The only precaution necessary is that the crystal should be uniformly illuminated in order that the exact position of extinction may be ascertained by use of one of the usual half-shadow stauroscopic plates.

The memoir concludes with a description of the general mode of investigating a biaxial crystal immersed in a liquid of equal refractive power, indicating how the principal planes of optical elasticity may be found, the positions of the optic axes ascertained, and the true internal angle of the latter measured. One of the most important advantages of the method is the simplification which it introduces into the study of triclinic crystals, hitherto almost dreaded by the crystallographer for the trouble they involve. It would appear that their optical investigation by the immersion method offers but slightly more difficulty than that of crystals of higher symmetry, the positions of the optic axes being readily found, and the true angle at once afforded. This alone would entitle Prof. Klein to the thanks of crystallographers and mineralogists for perfecting so admirable an aid to investigation,

A. E. TutTon.

\section{MICROBES AND METALS.}

$T \mathrm{HE}$ effect of metals on the growth of bacteria has been examined by Miller, Behring, and others, and another contribution to this subject has lately been published by Dr. Meade Bolton, in the December number of the International Medical Magazine. According to Uffelmann, who smeared the surface of copper coins with liquefied jelly-cultures of cholera bacilli, the latter were destroyed in seventeen minutes; whilst on a brass coin they were alive after thirty hours, but dead after sixty hours. Bolton employed Miller's method of inoculating a tube of melted jelly with particular microbes, and pouring the contents out on a sterilised glass-plate, after which bits of the metal under examination were laid on the jelly whilst it was still soft. If the metal has an inhibitory action on the microbes, then a clear zone is left around the metal after the colonies have developed in the other parts of the jelly. The width of this zone, Dr. Bolton found, varied very considerably with different organisms, as well as with different metals. Thus carefully purified bits of silver produced in the case of cholera bacilli a clear zone 5 millimetres broad, in the case of typhoid bacilli a zone of about I millimetre, whilst with the closely allied colon bacillus a zone of about 5 millimetres was produced. In the case of purified gold, no inhibition was observed with the staphylococcus pyogenes aureus, colon bacillus, typhoid bacillus, or cholera bacillus. Freshly "glowed gold" had invariably no inhibitory action; and in the few cases where inhibition was observed, the gold had not been glowed for several weeks. Pure nickel, platinum wire, and platinum black aluminium, silicon, and niobium, again, also failed to give any reaction with most of the microbes examined. Throughout the investigations it was found that those metals that are resistant towards chemical reagents in general, failed to produce any effect on microbes; whilst, on the other hand, those metals which are readily attacked by chemical reagents, all exhibited a marked inhibitory action upon the growth of bacteria. This result is probably due to a solution of the metal taking place in the medium. The length of time it is necessary to leave the metals in contact with the jelly, to produce an effect on the microbes present, was tried with brass, copper, cadmium, and zinc, on the staphylococcus pyogenes aureus. The metals were put on and removed at various intervals. When cadmium was left on for a minute, there was a clear space underneath where it had rested, which extended to I millimetre round; when it was left on for three or four minutes, the clear space usually extended over 3 millimetres. Very similar results were obtained with zinc. With brass no effect was produced when it was left on thirty-six minutes, but after this there was more and more marked inhibition up to fifty minutes; but to produce a clear space, it was necessary to leave it on for still longer. Copper produced no visible effect under thirty-six minutes, and fifty minutes was required to produce a clear space.

\section{G. C. Frankland.}

\section{PROFESSOR JAMES DWIGHT DANA.}

$\mathrm{B}^{\mathrm{Y}}$

the sudden death of Prof. J. D. Dana, from heartfailure, on April I 5, America has lost a veteran man of science, who in his time has not only played many widely varied parts, but has reached the highest excellence in each. As a mineralogist he published, solong ago. as I 837, the first edition of a "Descriptive Mineralogy," which by reason of its completeness and accuracy soon became a standard work of reference throughout the civilised world, and of which the sixth edition (II 34 pages), issued in 1892 under the superintendence of his distinguished son, Prof. Edward Salisbury Dana, still maintains the high reputation attained by the original work. As a geologist and palæontologist, he published in I 863 a similarly excellent and weil-illustrated "Manual of Geology," having special regard to the geology of the North American continent, and of which the fourth edition (1087 pages) was issued only two or three months ago. Of his work as a zoologist, we may cite as example his elaborate report on the zoophytes, collected by an expedition in which he took a very active part. The report is illustrated by $6 \mathrm{I}$ plates, and in it are described no fewer than 230 new species. Attainments so diverse belong only to the few.

James Dwight Dana was born on February 12, 1813, at Utica, in the State of New York, U.S.A., and was therefore in his eighty-third year at the time of his death. $\mathrm{He}$ was educated at Yale College, New Haven, Connecticut, receiving there a sound training in mathematics, physics and chemistry, which was of the greatest service to him in his subsequent career; he proceeded to his

No. 1330 , voL. 5 I] 